\title{
EUCOMMIA ULMOIDES OLIV. EXTRACT REGULATES AGES-INDUCED INJURY IN TUBULAR ENDOTHELIAL CELLS VIA THE RAGE-NRF2 PATHWAY
}

\author{
JINYOUNG HUR ${ }^{1,2}$, MOON HO DO ${ }^{1}$, MINA KIM ${ }^{1}$, JIWON CHOI ${ }^{1}$, YOONSOOK KIM ${ }^{1}$ \\ and SANG KEUN HA ${ }^{1,2 *}$
}

\author{
${ }^{1}$ Korea Food Research Institute, 245, Nongsaengmyeong-Ro, Iseo-Myeon, Wanju Gun, \\ Jeollabuk-DO 55365, Republic of Korea \\ ${ }^{2}$ Division of Food Biotechnology, University of Science and Technology, \\ Daejeon 34113, Republic of Korea
}

\begin{abstract}
The leaves, stems, and bark of Eucommia ulmoides Oliv. (EU), also known as Du-Zhong, have traditionally been used to cure various diseases, such as liver, kidney, and muscle diseases, in Asia. Despite evidence for protective effects against renal complications, its precise effects and mechanisms of action are unclear. In this study, the effects of EU on advanced glycation end products (AGEs)-induced renal disease and its mechanism were examined. NRK 52E normal rat kidney tubular epithelial cells were treated with AGEs and an EU extract. Expression levels of TGF- $\beta 1$, an indicator of renal cell damage, and catalase, an antioxidant marker, were examined. Nuclear factor-E2-related factor 2 (Nrf2), kelch-like ECH-associated protein 1 (keap1), and p65 regulation were examined to identify additional antioxidant mechanisms related to renal cell apoptosis and AGEs-induced renal cell damage. The effects of EU on mitogen-activated protein kinase (MAPK), Akt, and phosphoinositide 3-kinase (PI3K), which are involved in apoptosis, were also examined. TGF- $\beta 1$ expression increased in response to AGEs and decreased by additional treatment with EU. Additionally, EU increased the expression of catalase. We found that EU increased Nrf2, keap1, and p65 and regulated the expression of RAGE (receptor for AGEs) and its downstream target Sirt1. EU also regulated the AGEs-altered phosphorylation of apoptosis factors. Based on these findings, we concluded that EU regulates AGEs-induced renal cell damage via antioxidant and apoptosis-related mechanisms.
\end{abstract}

Keywords: diabetic nephropathy, advanced glycation end product, nuclear factor erythroid 2-related factor 2, Eucommia ulmoides

The pathologies of diabetes complications are diverse, and advanced glycation end products (AGEs) in foods or the body have recently been pinpointed as a direct cause of such complications. When the body maintains high blood sugar levels due to diabetes, proteins bind to sugars, which directly lead to diabetes complications and indirectly leads to metabolic disorders via elevated insulin resistance. AGEs that accumulate in the body induce various diabetes complications, such as diabetic retinopathy, diabetic neuropathy, and diabetic nephropathy (DN), as well as aging, arterial sclerosis, and Alzheimer's disease, by forming crosslinks with proteins or interacting with receptors for AGEs (RAGE), which stimulates inflammatory pathways and increases the release and apoptosis of cytokines (1). Recent studies of associations between AGEs and metabolic diseases, such as diabetes and obesity, have sparked extensive research on the development of metabolic diseases, such as insulin resistance and diabetes, caused by the distribution of food-derived AGEs in the body (2-4).

AGEs cause renal tissue injury by binding to RAGE, which leads to excessive ROS formation in renal tissues or cells, and by the generation of ROS and the profibrotic factor TGF- $\beta 1$ via nuclear factorkappa $\mathrm{B}(\mathrm{NF}-\kappa \mathrm{B})$ and mitogen-activated protein kinase (MAPK) (5-7). Therefore, the inhibition of AGEs-induced increases in TGF- $\beta 1$ and the reduction of ROS formation may be important aspects in the treatment of DN (8).

Nuclear factor-E2-related factor 2 (Nrf2) regulates toxic substances and oxidants and is an important factor in the defense against ROS. The Nrf2-

* Corresponding author: e-mail: skha@kfri.re.kr 
ARE pathway is the most important antioxidative system. During oxidative stress, Nrf2 is dissociated from kelch-like ECH-associated protein 1 (keap1) in the cytosol and migrates to the nucleus, where it targets genes that encode antioxidant proteins, such as heme-oxigenase1 (HO-1), catalase, glutathione peroxidase, and SOD1. The transcription of such genes not only defends against oxidative stress, but also protects against apoptosis, inflammation, and cancer $(9,10)$. Recent studies have explored the roles of Nrf2 activators, including single compounds or those derived from natural foods, in the prevention and treatment of diabetes and DN $(10,11)$.

Sirt1, a nicotinamide adenosine dinucleotide+ (NAD+) -dependent protein deacetylase belonging to the mammalian sirtuin family, responds to ROS stress (12). Despite recent studies of the roles of sirt1 in diabetes and its complications (13), the mechanisms by which Sirt1 regulates AGEsinduced nephropathy have not been investigated.

The leaves, stems, and bark of Eucommia ulmoides Oliv. (EU), also known as Du-Zhong, are traditionally used to cure various diseases, such as liver, kidney, and muscle diseases, in Asia. According to modern pharmacological studies, EU protects against hypertension, hyperglycemia, diabetes, obesity, osteoporosis, Alzheimer's disease, aging, and sexual dysfunction $(14,15)$. Some studies have investigated the protective effects of EU against renal complications, but its specific effects on AGEs-induced DN are unclear. NRK-52E cells are rat renal proximal tubular cells used in research on renal cell damage and relevant therapeutic drugs. In this study, we investigated whether EU could inhibit TGF- $\beta 1$ and increase catalase expression by promoting the activation of the Nrf2-ARE pathway in AGEs-induced NRK-52E cells. Here, we showed that EU significantly increased the transcriptional activity of Nrf2 and thereby upregulated the expression of Nrf2 target genes, including catalase. Importantly, the EU-induced activation of the AGEs-RAGE-Nrf2 anti-oxidative pathway decreased AGEs-induced TGF- $\beta 1$ and, as a consequence, slowed the progression of DN.

\section{Materials and Methods Materials}

Dimethyl sulfoxide, Geniposidic acid (GA), pinoresinol diglucoside and formic acid were purchased from Sigma Aldrich (St. Louis, MO, USA). Cell culture media [Dulbecco's modified Eagle's medium (DMEM)], bovine serum (BS), and other ingredients required to culture cells were supplied by Life Technologies (Grand Island, NY, USA). A
TGF-1 $\beta$ Immunoassay Elisa Kit was obtained from RnD Systems (Minneapolis, MN, USA). The Nuclear/Cytosol Fraction Kit was obtained from Biovision (Milpitas, CA, USA). RAGE, Sirt1, catalase, p38, p-p-38, Extracellular signal-regulated kinases (ERK), p-ERK, c-Jun N-terminal kinases (JNK), p-JNK, Akt, p-Akt, phosphoinositide 3kinase (PI3K), p-PI3K and Nrf2 were purchased from Cell Signaling Technology (Danvers, MA, USA) and Lamin B, p-p65, p-65 and $\beta$-actin, and secondary antibodies were purchased from Santa Cruz Biotechnology (Santa Cruz, CA, USA).

\section{Preparation of extract of Eucommia ulmoides Oliv}

Eucommia ulmoides Oliv. was purchased from Kyungdong market in Seoul, Korea and a voucher specimen (H-378) of the herb was deposited in the Division of Functional Food Research, Korea Food Research Institute. Whole plants of E. ulmoides Oliv. (100 g) was subjected to extraction with $70 \%$ ethanol, and the extracts were evaporated on a rotary vacuum evaporator. The filtrate was lyophilized using a freeze-dryer and stored at $-20^{\circ} \mathrm{C}$ until use. The yield of Eucommia ulmoides Oliv. was $11.5 \%$.

\section{Preparation and characterization of glyceralde- hyde-induced AGEs}

Bovine serum albumin (BSA, $20 \mathrm{mg} / \mathrm{mL}$ ) was incubated with $20 \mathrm{mM}$ glyceraldehyde in $0.1 \mathrm{M}$ potassium phosphate-buffered saline (PBS, pH 7.4) containing $1 \mathrm{mM}$ diethylene triamine pentaacetic acid for $7 \mathrm{~d}$ at $37^{\circ} \mathrm{C}$ in the dark. Control non-glycated BSA was incubated in the same conditions, but without reducing sugars. The AGEs content was estimated by spectrofluorometry with an excitation wavelength of $370 \mathrm{~nm}$ and an emission wavelength of $440 \mathrm{~nm}$, revealing a 200-fold increase in fluorescence for glyceraldehyde-AGEs compared to control BSA.

\section{UPLC-MS/MS analysis}

The analyses were performed using an Acquity UPLC system (Waters, Milford, MA, USA) with an Acquity UPLC BEH C18 column $(2.1 \mathrm{~mm} \times 100$ $\mathrm{mm}, 1.7 \mu \mathrm{m})$. The mass spectrometer was a Waters Xevo TQ triple-quadrupole mass spectrometer equipped with electrospray ionization (ESI) source. MassLynx 4.1 (Waters) was used for data processing. The mobile phase included $0.1 \%$ formic acid aqueous solution (Solvent $\mathrm{A}$ ) and $0.1 \%$ formic acid in acetonitrile (Solvent B) and the following gradient elution program was performed: 0-3 min, 95-50\% solvent A; 3-4.5 min, 50-20\% solvent A; 4.5-5 min, 20-5\% solvent A; 5-5.5 min, 5\% solvent 
A; 5.5-6.5 min, 5-95\% solvent A. The total run time was $7 \mathrm{~min}$. The column temperature was kept at $25^{\circ} \mathrm{C}$ and the flow rate was set at $0.3 \mathrm{~mL} / \mathrm{min}$. The auto-sampler was conditioned at $4^{\circ} \mathrm{C}$ and the injection volume was $3 \mu \mathrm{L}$. The LC-MS/MS system was operated in negative ESI mode and scanned using multiple reaction monitoring (MRM) mode. The MRM transitions were monitored at $\mathrm{m} / \mathrm{z} 373.1 \rightarrow \mathrm{m} / \mathrm{z}$ 211.1 for geniposidic acid $\mathrm{A}$ and $\mathrm{m} / \mathrm{z} 681.1 \rightarrow \mathrm{m} / \mathrm{z}$ 357.0 for pinoresinol diglucoside. The voltage of the capillary, cone and collision energy were set at 3.2 $\mathrm{kV}, 34 \mathrm{~V}$, and $22 \mathrm{~V}$, respectively. The gas flow for desolvation and the cone were 800 and $50 \mathrm{~L} / \mathrm{h}$. The source temperature and desolvation gas temperature were $150^{\circ} \mathrm{C}$ and $400^{\circ} \mathrm{C}$, respectively.

\section{Cell culture and treatments}

NRK 52E normal rat kidney tubular epithelial cells were obtained from the ATCC (Manassas, VA, USA) and maintained in DMEM supplemented with $5 \%$ in a humidified incubator with $5 \% \mathrm{CO}_{2}$ at $37^{\circ} \mathrm{C}$. EU was dissolved in $1 \%$ of dimethyl sulfoxide to a final concentration of $100 \mathrm{mg} / \mathrm{mL}$ for the stock solution and used at various concentrations by dilution in serum-free media. Moreover, glyceraldehydeAGEs were obtained as a $10 \mathrm{mg} / \mathrm{mL}$ stock solution and used at various concentrations by dilution in serum-free media.

\section{TGF-1 $\beta$ Immunoassay}

TGF-1 $\beta$ expressions were determined by an enzyme-linked immunosorbent assay (ELISA). NRK 52E cells were seeded in a 96-well plate and treated with AGEs, EU, and a combination of AGEs and EU for $24 \mathrm{~h}$. The conditioned media were analyzed using a TGF-1 $\beta$ Immunoassay Kit as per the manufacturer's instructions (RnD Systems (Minneapolis, MN, USA).

\section{Western blot analysis}

NRK 52E cells were incubated with EU in the absence or presence AGEs and lysed with Protein
Extraction Solution. The same amount of protein from each sample was separated by $10 \%$ SDSPAGE gel transferred onto a nitrocellulose membrane. The membranes incubated with primary antibodies against RAGE, Sirt1, catalase, p-p65, p-65, p-p38, p-38, p-ERK, ERK, p-JNK, JNK, p-Akt, Akt, p-PI3K, PI3K, Nrf2, Lamin B, and $\beta$-actin. Antibody detection was performed using ECL reagent (Thermo Scientific) and visualized by ChemiDoc (Bio-Rad Laboratories, Hercules, CA, USA). The intensities of the bands were normalized to the $\beta$-actin or Lamin B band using Image Lab (Bio-Rad).

\section{Statistical analysis}

All statistical parameters were calculated using GraphPad Prism 3.0 (GraphPad Software Inc., San Diego, CA, USA). Values are expressed as means \pm standard error of the mean (SEM). The results were analyzed by one-way analysis of variance (ANOVA) followed by Tukey's post-hoc tests. Differences with a $p$-value of less than 0.05 were considered statistically significant.

\section{RESULTS}

\section{Geniposidic acid concentration in EU}

The previous study reported that the main component of EU extract is iridoid glycosides (14). Among these compounds, GA is well-known to possess various pharmacological effects. Based on a UPLC-MS/MS analysis, we revealed the concentration of GA in EU to be $77.0 \pm 0.26 \mathrm{mg} / \mathrm{g}$ (Fig. 1).

\section{EU increased catalase and decreased TGF- $\beta 1$ in AGEs-induced NRK-52E cells}

We examined the effects of EU on AGEsinduced TGF- $\beta 1$ and catalase expression in NRK52E cells. In particular, NRK-52 renal tubular epithelial cells were treated with $100 \mu \mathrm{g} / \mathrm{mL}$ AGEs, and TGF- $\beta$ protein levels increased by about twofold, as determined by ELISA. Treatment with

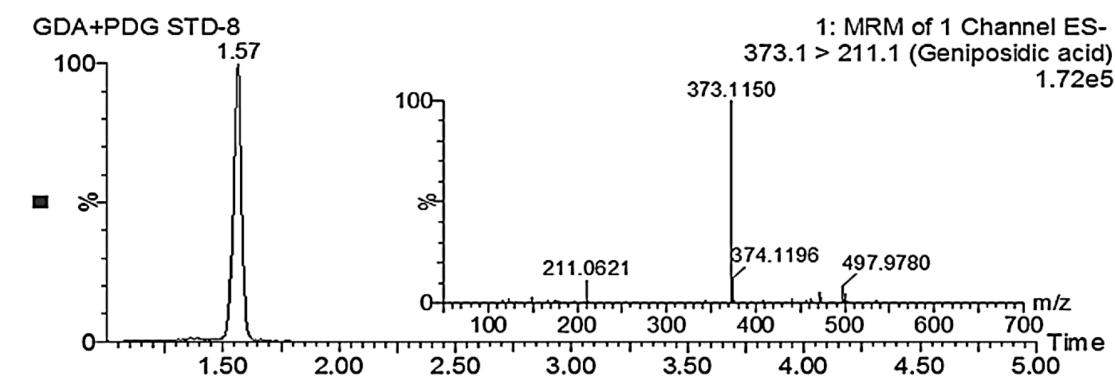

Figure 1. UPLC-MS/MS chromatogram of Eucommia ulmoides Oliv. extracts 
10, 50, and $100 \mu \mathrm{g} / \mathrm{mL}$ EU led to dose-dependent reductions in these levels (Fig. 2A). Further, based on western blotting, the expression of catalase, was significantly reduced after treatment with AGEs, however, increased in a dose-dependent manner in EU treatment groups (Fig. 2B).

EU regulates the Nrf2 pathway and reduces RAGE expression.

We treated NRK-52E cells with EU extracts and confirmed that Nrf2 expression increased proportionately with the EU concentration in the nuclear fraction. In particular, Nrf2 expression was greater than 1.5 times higher in cells treated with $100 \mu \mathrm{g} / \mathrm{mL}$ than in untreated cells. Keap1 expression exhibited the opposite trend to that of Nrf2, confirming that the EU extract regulates catalase and other antioxidant factors by regulating $\mathrm{Nrf} 2$. Moreover, RAGE, an AGEs receptor, has been reported to increase in response to treatment with AGEs in a concentration-dependent manner in diabetic conditions. We verified that RAGE expression increased in response to AGEs in NRK-52E cells and was dose-dependently reduced by treatment with the EU extract and elevated sirt1 expression; we speculate that the EU extract influences downstream RAGE signaling by increasing sirt1 expression, a downstream factor (Fig. 3).

\section{EU regulates the ROS-related signaling and MAPK-Akt pathway}

We examined the expression of NF- $\mathrm{KB}$, which acts downstream of AGEs and is an important factor in inflammation and oxidative stress. Elevated p65

A

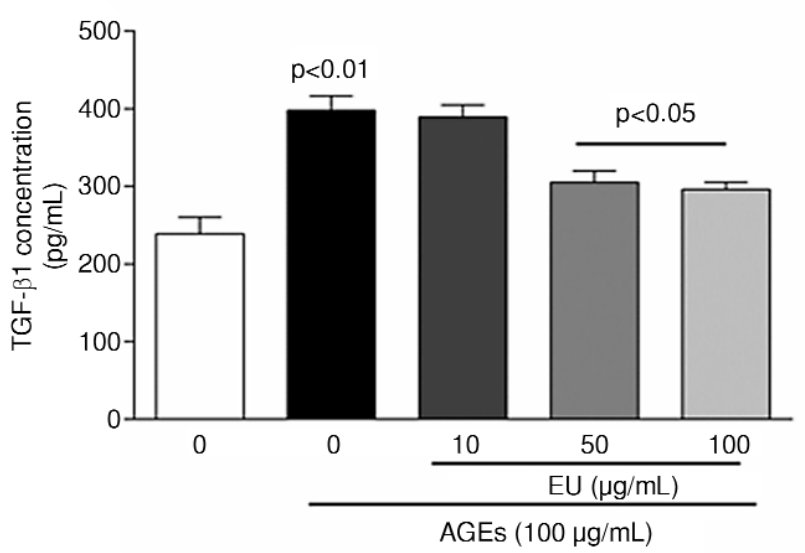

B

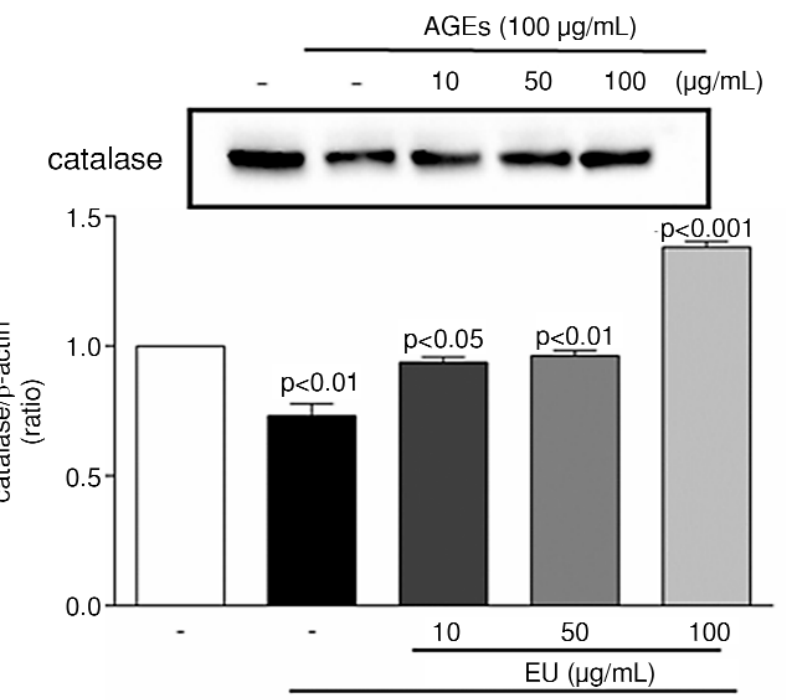

AGEs $(100 \mu \mathrm{g} / \mathrm{mL})$

Figure 2. EU decreased TGF $\beta 1$ expression and increased catalase expression in AGEs-induced NRK-52E cells. After treatment with various concentrations of $\mathrm{EU}(10,50$, and $100 \mu \mathrm{g} / \mathrm{mL})$ and AGEs $(100 \mu \mathrm{g} / \mathrm{mL})$ for $24 \mathrm{~h}$, the protein was extracted and subjected to ELISA (A) and western blot assays (B). Data are presented as means \pm SEM of 3 independent experiments 
A
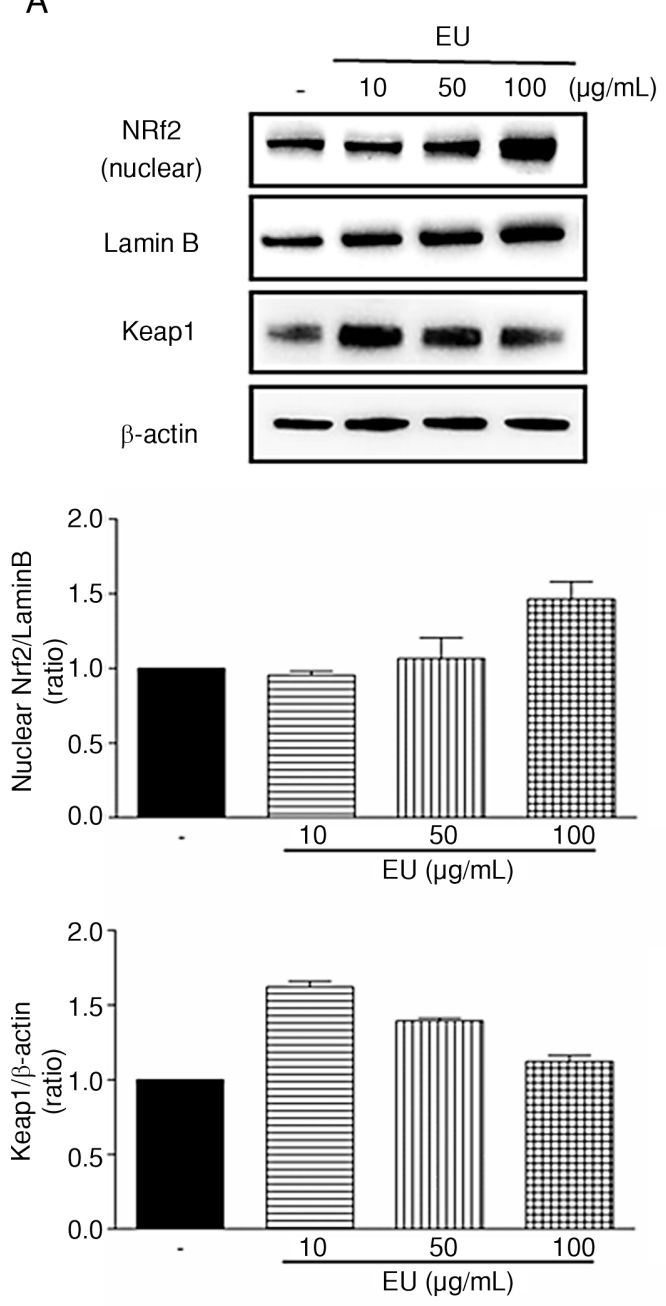

B
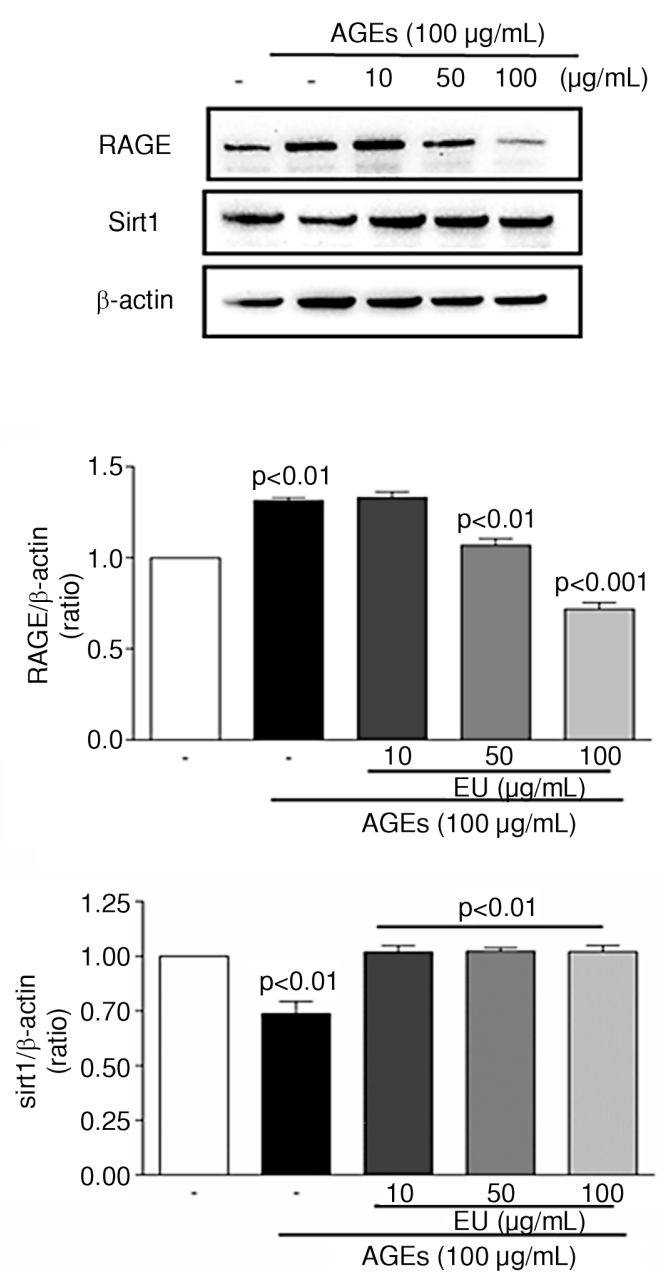

Figure 3. Effect of EU on Nrf2 (A) and RAGE expression (B). Cells were incubated with EU for the indicated time and cytosolic and nuclear fractions were prepared for Nrf2 analysis. Protein expression levels of Keap1, Nrf2, RAGE, and Sirt1 were normalized to $\beta$-actin levels in the cytosolic fraction and lamin B levels in nuclear fractions. Data are presented as means \pm SEM of 3 independent experiments

phosphorylation was observed in response to the treatment of NRK-52E cells with AGEs, and this elevation was significantly and dose-dependently decreased after treatment with the EU extract. The opposite trend was evident in the cytosolic fraction. These results suggest that the EU extract regulates RAGE expression and affects the phosphorylation of NF- $\mathrm{BB}$, thereby inhibiting oxidative stress. To examine renal cellular dysfunction as a result of oxidative stress as well as the inhibition of cellular apoptosis caused by renal cytotoxicity, we investigated how EU regulates MAPK and Akt activation. The phosphorylation of $\mathrm{p} 38$, a MAPK related to cellular stress, was increased by about 1.4 times by AGEs but returned to the levels observed in the control after treatment with the EU extract. There were no significant effects of EU on JNK phosphorylation. Akt phosphorylation was significantly reduced by AGEs and dose-dependently increased by the EU extract; in particular, it increased by 1.3 times by treatment with $100 \mu \mathrm{g} / \mathrm{mL}$ compared with levels in control cells. The effects of EU on PI3-kinase were similar to those observed for Akt phosphorylation (Fig. 4).

\section{DISCUSSION}

In this study, we showed that EU extracts inhibit cellular injuries induced by AGEs in rat tubular epithelial cells via a downstream mechanism involving the AGEs-RAGE axis. AGEs are formed via the Maillard reaction, which involves a non- 
enzymatic condensation reaction between the carbonyl group of the reducing sugar and 1́-amino group in proteins in foods. In the body, AGEs form when proteins bind to sugars in diabetic conditions with high blood sugar levels, directly causing diabetic complications and indirectly inducing metabolic disorders caused by elevated insulin resistance (3). The pathologies of diabetic complications vary widely, and AGEs in foods and the body are direct causes of these complications.

Recent studies of the association between AGEs and metabolic diseases, such as diabetes and obesity, have prompted extensive research focusing on the development of metabolic diseases, including insulin resistance and diabetes, caused by the distribution of food-derived AGEs in the body (2). The generation and accumulation of AGEs are increased in diabetes and diabetic complications, particularly in DN, and AGEs accumulate across various tissues. Diabetic complications can develop in both Type 1 and Type 2 diabetes, and many reports have con- firmed that patients with diabetic complications show AGEs accumulation in tissues and serum (16). Thus, it is believed that diabetic complications may be regulated by the inhibition of AGEs accumulation or mechanisms downstream of accumulated AGEs in relevant cells. In the present study, we verified the cytoprotective effects of EU in AGEsinduced rat kidney tubular epithelial cells (NRK52E). E ulmoides Oliv., also known as Du-Zhong, is a traditional herb that has long been used in Chinese and Korean medicine (13). In this study, a $70 \%$ EtOH EU extract was used. Twenty-four iridoids have been isolated and identified from EU, and among them, geniposide reported to has anti-tumor activity $(14,17,18)$. We measured the geniposide content by LC/MS and we plan to perform physiological analyses to verify these findings in the future study. TGF $\beta$ is a profibrotic cytokine and a causal factor in diabetic neuropathy. TGF- $\beta 1$ increases the production of cellular matrix by renal cells and directly affects the expression of its transcription
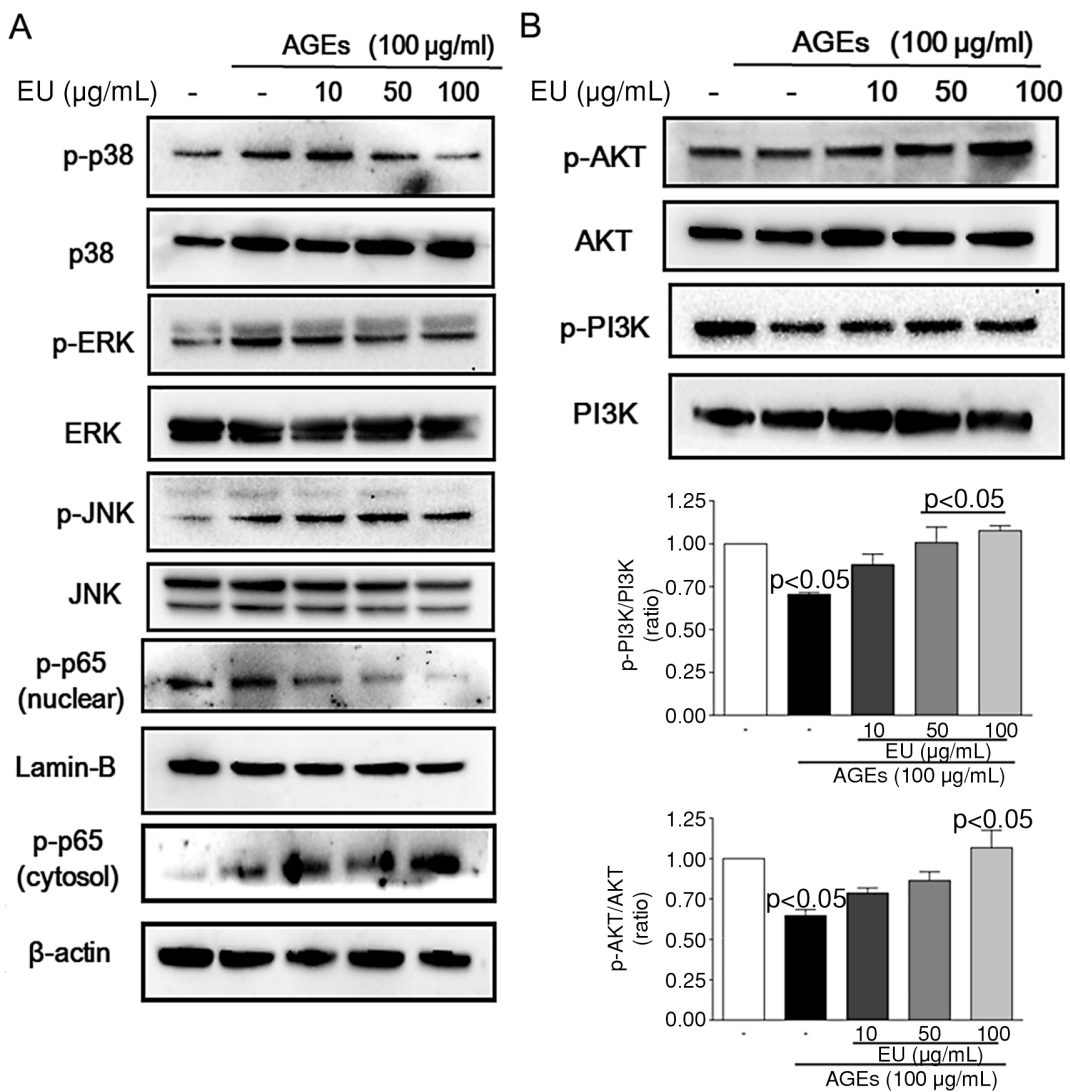

Figure 4. Effects of EU on MAPK, Akt phosphorylation and NF- $\kappa B$ p65 translocation in AGEs-stimulated NRK-52E cells. After 30-min treatment with AGEs $(100 \mu \mathrm{g} / \mathrm{mL})$ in the presence or absence of EU $(10,50$, and $100 \mu \mathrm{g} / \mathrm{mL})$, cell lysates were prepared for western blot analyses. Protein expression levels of p-ERK, p-p38, p-JNK and NF- $\mathrm{BB}$ p65 (A) and p-Akt and p-PI3K (B) were normalized to levels of the total form. Representative bands from individual experiments are shown in (A) and (B). Data are presented as means \pm SEM of 3 independent experiments 
factor (19). The elevation of TGF- $\beta 1$ in AGEsinduced NRK-52E cells indicates damage to NRK-52 cells, as reported in the literature (20). Considering that EU decreasing TGF- $\beta 1$ expression, it may inhibit injuries in tubular epithelial cells, such as NRK-52E cells. RAGE, a membrane of the immunoglobulin superfamily, is activated by various ligands, resulting in the inhibition of apoptosis and phosphorylation of MAPK by PI3-Kinase activation; the activated factors regulate transcription factors, such as NF- $\mathrm{KB}$ and $\mathrm{Nrf} 2$, thereby generating pro-inflammatory cytokines or factors that affect oxidative cell damage (21). Furthermore, owing to a recent report that shed light on the elevation of RAGE expression in various diseases, there is a great deal of interest in the regulation of RAGE, its downstream targets, and diseases. We found that the treatment of NRK-52E cells with AGEs increased RAGE expression, and EU reduced the elevated RAGE expression. Furthermore, we examined the phosphorylation of PI3K and Akt, which are downstream factors, and found that reduced levels of phosphorylation in response to AGEs were increased by treatment with EU. We also measured the activity of MAPK, another regulator, and found that p-p-38 and p-ERK expression levels were inhibited, but there were no changes in the phosphorylation of JNK. We examined the activity of NF- $\mathrm{KB}$, a regulator of ROS expression that induces various diseases, and found that EU may defend against oxidative stress caused by AGEs in NRK-52E cells by reducing phosphorylation in the nuclear fraction. Sirt1 is a very important intracellular functional protein and is regulated at the transcriptional level and by various chemicals. Recent studies have reported that sirt1 expression is reduced in the renal tissues of diabetic $\mathrm{db} / \mathrm{db}$ mice and high glucose-induced mice models (22), and studies are underway to explore the important roles of activators that regulate sirt1 in the treatment of DN (23). Our findings indicated that EU increases Sirt1 expression after reductions by AGEs, confirming that EU also has an impact on sirt1 expression.

The Nrf2-Keap1 system is one of the most important cytoprotective mechanisms. Nrf2 is bound to keap1, a cytosolic repressor protein, in the cytosol; it is dissociated from Keap1 once the cell is exposed to stress, such as ROS. Nrf2 in the nucleus is heterodimerized with transcription factors, such as small Maf proteins, and binds to the antioxidant response element (ARE) or a target gene promoter to induce the transcription of antioxidant genes. Oxidative stress and inflammation are important mediators of the symptoms and progression of chronic kidney disease. Activation of Nrf2 maintains the redox balance in normal conditions and stimulates the expression of various antioxidant factors to defend against ROS. In light of this, recent studies have aimed to develop Nrf2 activators as therapeutic agents for chronic kidney disease or resulting $\mathrm{DN}$ (7, 24-26). In this study, we also attempted to verify whether EU acts as an Nrf2 activator in renal cells with AGEs-induced damage. We confirmed that Nrf2 was reduced by AGEs in the nuclear fraction and that the reduction was dosedependently reversed by EU, suggesting that EU acts as an Nrf2 activator and that it can inhibit ROSinduced renal cell injury. Further, keap1 expression decreased dose-dependently in the cytosolic fraction, indicating that Nrf2/keap1 is dissociated as a defense mechanism in response to external stimuli. These findings point to a new material with protective effects against AGEs-induced renal cell damage. In particular, they suggest that EU is a promising therapeutic and protective agent for DN by functioning as an Nrf2 activator and thereby defending against the AGEs-RAGE system and ROS. Additional studies are needed to investigate EU and its bioactive substances as well as other mechanisms by which EU inhibits renal cell damage. These results extend our knowledge of the role of EU in the prevention of AGEs-induced renal complications and provide a basis for the development of therapeutic agents.

\section{CONCLUSION}

In this study, we confirmed that EU inhibits damage caused by AGEs in rat kidney tubular epithelial cells. EU regulated AGEs-RAGE signaling and, in particular, increased the expression of Nrf2-Keap, which defends against oxidative stress. Furthermore, by regulating the phosphorylation of factors related to apoptosis, i.e., MAPK and AktPI3-Kinase, it alleviated damage in rat kidney tubular epithelial cells. These results extend our knowledge of the role of EU in the prevention of AGEsinduced renal complications and provide a basis for the development of therapeutic agents.

\section{Acknowledgment}

This work was supported by the Main Research Program E0164403 of the Korea Food Research Institute (KFRI) funded by the Ministry of Science.

\section{Conflict of interest}

All authors declare that they have no competing financial interests. 


\section{REFERENCES}

1. Gasser A., Forbes J.M.: Protein Pept. Lett. 15, 385 (2008).

2. Uribarri J., Woodruff S., Goodman S., Cai W., Chen X. et al.: J. Am. Diet. Assoc. 110, 911 (2010).

3. Bohlender J.M., Franke S., Stein G., Wolf G.: Am. J. Physiol. Renal Physiol. 289, F645 (2005).

4. Daroux M., Prevost G., Maillard-Lefebvre H., Gaxatte C., D'Agati V.D. et al.: Diabetes Metab. 36, 1 (2010).

5. Kashihara N., Haruna Y., Kondeti V.K., Kanwar Y.S.: Curr. Med. Chem. 17, 4256 (2010).

6. Singh D.K., Winocour P., Farrington K.: Nat. Rev. Endocrinol. 7, 176 (2011).

7. Yamagishi S., Matsui T., Oxid. Med. Cell Longev. 3, 101 (2010).

8. Lam S., van der Geest R.N., Verhagen N.A., Daha M.R., van Kooten C.: Nephrol. Dial. Transplant. 19, 1694 (2004).

9. Kensler T.W., Wakabayashi N., Biswal S.: Annu. Rev. Pharmacol. Toxicol. 47, 89 (2007).

10. Zoja C., Benigni A., Remuzzi G.: Nephrol. Dial. Transplant. 29 Suppl 1, i19 (2014).

11. Jimenez-Osorio A.S., Gonzalez-Reyes S., Pedraza-Chaverri J.: Clin. Chim. Acta 448, 182 (2015).

12. Chong Z.Z., Shang Y.C., Wang S., Maiese K.: Expert Opin. Ther. Targets 16, 167 (2012).
13. Lee J.H., Song M.Y., Song E.K., Kim E.K., Moon W.S. et al.: Diabetes 58, 344 (2009).

14. He X., Wang J., Li M., Hao D., Yang Y., Zhang C. et al.: J. Ethnopharmacol. 151, 78 (2014).

15. Zhou Y., Liang M., Li W., Li K., Li P. et al.: Environ. Toxicol. Pharmacol. 28, 342 (2009).

16. Wang W., Wang Y., Long J., Wang J., Haudek S.B. et al.: Cell Metab. 15, 186 (2012).

17. Nakamura T., Nakazawa Y., Onizuka S., Satoh S., Chiba A. et al.: Mutat. Res. 388, 7 (1997).

18. Li Y., Sato T., Metori K., Koike K., Che Q.M., Takahashi S.: Biol. Pharm. Bull. 21, 1306 (1998).

19. Ketteler M., Noble N.A., Border W.A.: Curr. Opin. Nephrol. Hypertens. 3, 446 (1994).

20. Tian L., Fu P., Zhou M., Gu Y., Li Y., Qi J.: Int. J. Mol. Med. 38, 1831 (2016).

21. Lukic I.K., Humpert P.M., Nawroth P.P., Bierhaus A.: Ann. N. Y. Acad. Sci. 1126, 76 (2008).

22. Kim M.Y., Lim J.H., Youn H.H., Hong Y.A., Yang K.S. et al.: Diabetologia 56, 204 (2013).

23. Li C., Cai F., Yang Y., Zhao X., Wang C. et al.: Eur. J. Pharmacol. 649, 382 (2010).

24. Jung K.A., Kwak M.K.: Molecules 15, 7266 (2010).

25. de Haan J.B.: Diabetes 60, 2683 (2011).

26. Zheng H., Whitman S.A., Wu W., Wondrak G.T., Wong P.K. et al.: Diabetes 60, 3055 (2011).

Received: 10.01.2019 